\title{
ALL FLEXIBLE GRAPHENE STRAIN SENSORS WITH LIQUID METAL INTERCONNECTORS FOR EMBEDDED STRUCTURAL HEALTH MONITORING AND SKIN TACTILE SENSING
}

\author{
Yueyi Jiao, Clinton Young, Shuo Yang, Ceylan Halil, and Liang Dong*
}

Iowa State University, Ames, Iowa, USA

\begin{abstract}
This paper reports on the development of graphene strain sensors made of all flexible materials, including graphene, liquid metal, and elastomer. The sensors are featured with a flexible interconnector design using liquid metal as an interconnecting material for graphene-based sensing elements. This design allows enhancing flexibility and reliability of the sensors compared with existing hard metal based interconnection methods. A unidirectional strain sensor and a multidirectional rosette-type strain sensor are developed by encasing patterned graphene and liquid metal in an elastomer. We explore the applications of the unidirectional sensor for structural health monitoring by attaching the sensor on the surface of concrete, and for tactile sensing by attaching the sensor on human skin. This work represents an exploratory effort on using liquid metal interconnectors for graphene-based sensors to obtain full structural flexibility.
\end{abstract}

\section{INTRODUCTION}

Strain sensors are used to monitor structural damage, evaluate material fatigue properties, and track human body movement. Recently, graphene has been investigated as a strain sensing material due to its high piezoresistivity, carrier mobility, and Young's modulus [1-3]. Significant efforts have been made to develop various graphene-based flexible strain and pressure sensors [4-8]. While these existing graphene-based sensors are regarded as flexible devices, electrical interconnections for these devices are generally obtained using hard metal/alloy materials [9]. This has compromised overall structural flexibility of the devices and has caused a long-term reliability issue due to possible local strains introduced to the interconnecting areas during sensing and/or actuation.

Many liquid metals and their alloys are found to be non-toxic, non-evaporative, and melt at room temperature. They usually have comparable electrical conductivities and better wetting properties compared with mercury [10]. As an electrical interconnecting material, liquid metal has been used in skin type temperature and pressure sensors with a mixture of PDMS and carbon nanotubes [11-14], and also has been reported to accommodate thermal stresses and provide re-workability in case of chip failure or upgrade [15]. In addition, liquid metals have been used as electrical interconnects for integrated circuits to overcome some packaging challenges in wire bonding and flip-chip bonding [16].

In this paper, we reports flexible graphene strain sensors using liquid metal as interconnectors for enhanced structural flexibility and reliability. The sensors are formed by encasing graphene sensing elements in an elastomer with built-in microfluidic channels filled with liquid metal. By integrating graphene and liquid metal in an elastomer, the sensor gains full structural flexibility. By stacking multiple flexible sensors layer by layer together with a rosette structure, the obtained rosette sensor can measure strains coming from multiple directions. To explore potential applications of these sensors, we demonstrate feasibility to monitor strain of concrete using a surface attached unidirectional sensor, and to track motion behaviors of human body using a sensor attached on human skin.

\section{DEVICE FABRICATION}

Three major steps are involved in fabricating graphene sensors, including patterning and transferring of graphene, formation of microfluidic channel, and formation of liquid metal interconnectors (Fig. 1). First, photoresist was spin coated on the surface of a nickel foil deposited with $\sim 105 \mathrm{~nm}$ thick graphene, with $500 \mathrm{rmp}$ for $5 \mathrm{sec}$ and then $4000 \mathrm{rmp}$ for $45 \mathrm{sec}$. Subsequently, photolithography was used to form patterns in photoresist. Then, oxygen plasma was used to etch away unwanted graphene from the nickel foil, with the etching time of 50 mins. After that, polydimethylsiloxane (PDMS, Sylgard, 15:1 part A:B) was degassed to remove bubbles. $15 \mathrm{~g}$ of the PDMS precursor was then poured into a 3 inch diameter petri-dish and baked at $85{ }^{\circ} \mathrm{C}$ for $40 \mathrm{~min}$. The cured PDMS slab was cut and peeled out of the petri dish and then immediately stamped onto the fresh surface of the patterned graphene. Then, the entire substrate was immersed into $5 \mathrm{wt} \% \mathrm{FeCl}_{3}$ solution for 24 hours or until the nickel foil was fully etched.

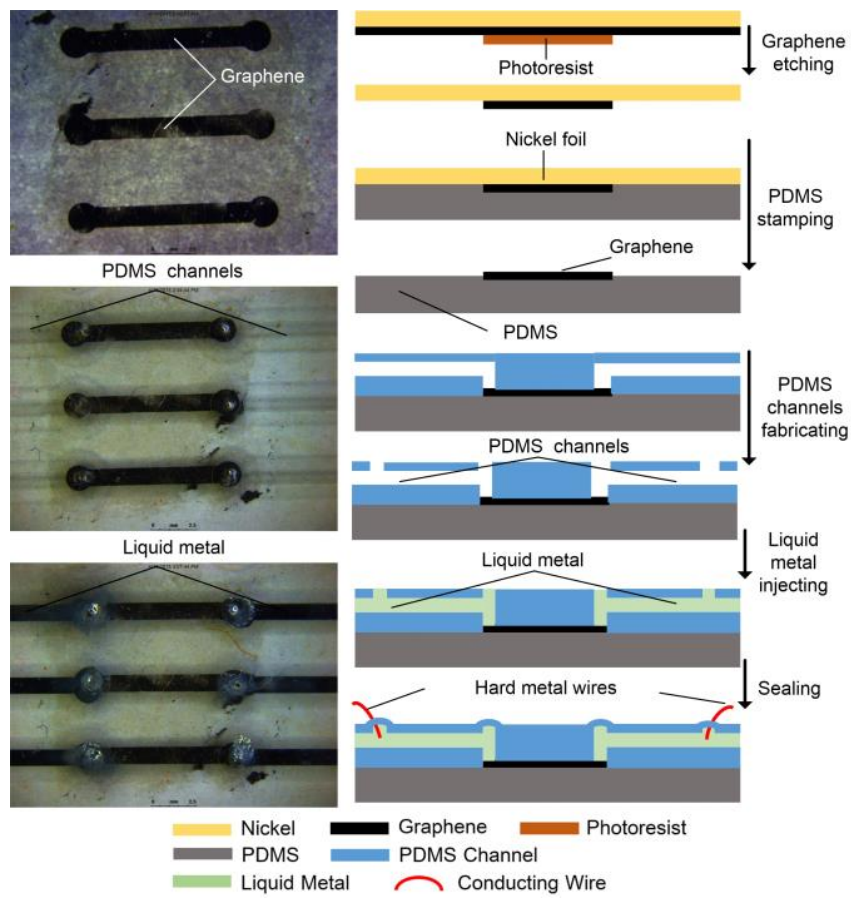

Figure 1: Process flow for an array of graphene-based strain sensors. The process mainly involves embedding patterned graphene into PDMS, and flowing liquid metal into microfluidic channels to realize interconnection.

In order to allow liquid metal to access and directly contact the patterned graphene, microfluidic channels were formed by soft lithography. In this process, photoresist patterns were fabricated on a glass slide as a master mold, followed by pouring PDMS solution (Sylgard, 15:1 part A: B) and baking at $70{ }^{\circ} \mathrm{C}$ for $40 \mathrm{~min}$. After the PDMS layer with patterned channels was peeled off from the mold, accessing holes were manually punched through the PDMS channel layer. Subsequently, the PDMS channel layer and the previously formed PDMS layer with graphene patterns were bonded with the 
help of oxygen plasma treatment. Then, Gallium-Indium eutectic or EGaIn was injected into the channels using syringe needles. Here, EGaIn is considered as a promising substitution of mercury because of its liquid state in room temperature and low toxicity. The liquid metal was able to reach and cover the contact pads of the graphene patterns. Lastly, metal wires were inserted into the channels through the accessing holes to realize electrical connections to external circuits and these accessing holes were sealed by thermally curing PDMS precursor solution dropped at the holes.

By using this fabrication method, a graphene based strain sensor capable of detecting strain in a single direction was formed. A multidirectional rosette type strain sensor (Fig. 2) employed three unidirectional strain sensors arranged with 60 degrees angle between neighboring sensors. To reduce the total thickness of the rosette sensor, instead of simply stacking three unidirectional graphene strain sensors vertically, the PDMS layer for the liquid metal channels and the other PDMS layer for the patterned graphene were combined together. In other words, the PDMS layer containing the liquid metal channels which conduct strain signals for the lowest layer graphene, also served as the middle layer graphene substrate.

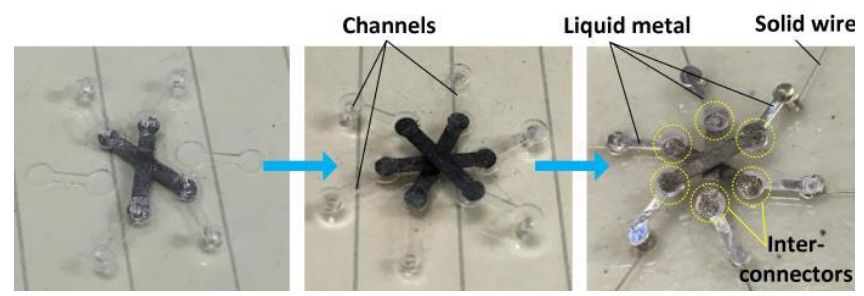

Figure 2: Process flow of a stacked rosette strain sensor

\section{RESULTS AND DISCUSSION}

Due to the flexible nature of silicone, liquid metal, and graphene, the sensors could be deformed into different shapes without sacrificing durability, conductivity, and robustness of the sensors (Fig. 3). For example, mechanical twisting or bending at the sensing area did not affect electrical interconnection between liquid metal and graphene patterns.

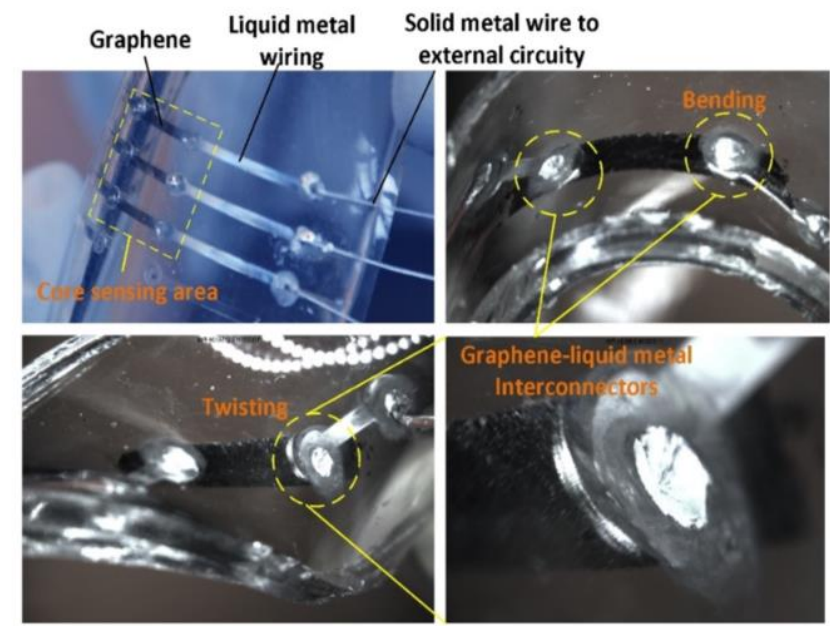

Figure 3: Photos showing interconnecting, external wiring, bending, and twisting of the graphene-liquid metal strain sensors.

Different tensile strains were applied to the sensor by stretching the sensor using a motored linear stage. The resistance value of the sensor at a given strain was recorded when it became stable. By releasing the sensor to its starting zero strain state, the resistance of the sensor went back to its initial value. Fig. 4a shows the resistance is varied between $\sim 245$ and $\sim 295 \Omega$ as the applied strain increases up to $9 \%$.

Fig. $4 \mathrm{~b}$ shows the resistance responses of both liquid metal and graphene elements to the same levels of applied strains. We used gauge factor to evaluate how sensitive the two elements responded to applied strains. Here, gauge factor is defined as $(\mathrm{dR} / \mathrm{R}) /(\mathrm{dL} / \mathrm{L})$, where $\mathrm{R}$ and $\mathrm{L}$ represent the resistance and length of the sensor, respectively. The results demonstrate that the gauge factor of the graphene element of the sensor is $\sim 1.5$. As the strain increased, the resistance of the sensor grew in a linear fashion. However, due to possessing an almost zero gauge factor, the liquid metal element of the sensor contributed little to the total response of the sensor to applied strain.

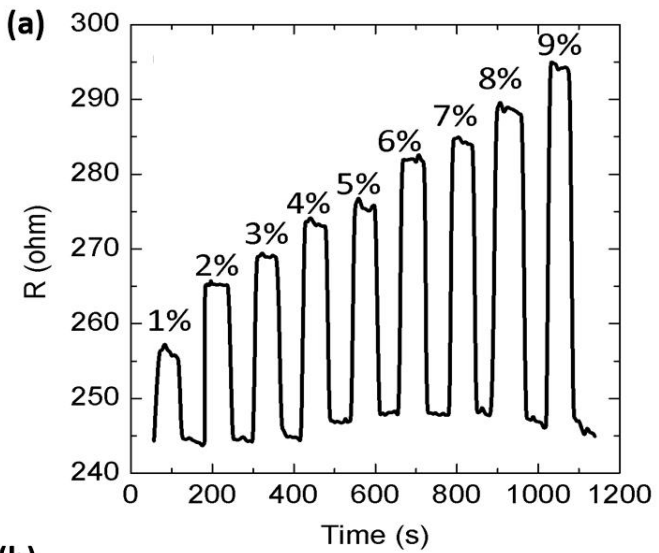

(b)

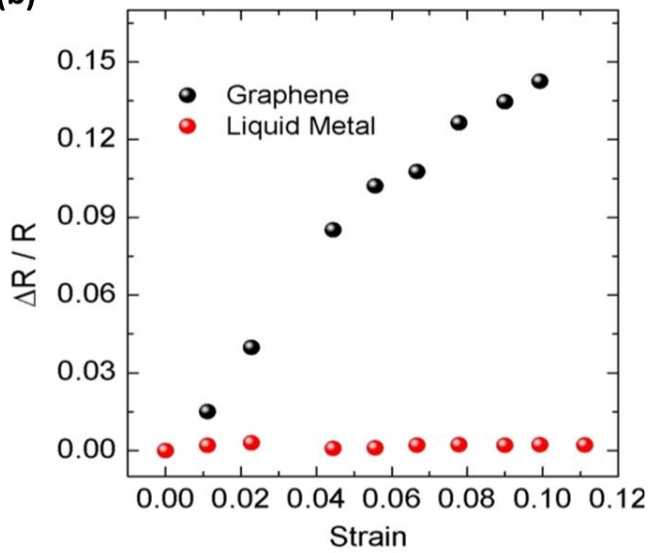

Figure 1: (a) Resistance response of the unidirectional strain sensor to applied strains during the cyclic stretch and release measurement. (b) Relative resistance change of the graphene sensor as a function of applied strain.

For the multidirectional rosette sensor (Fig. 5a), the normal strain $\varepsilon_{\theta}$ at any angle $\theta$ from the major principal axis can be expressed as [17]:

$$
\varepsilon_{\Theta}=\frac{\varepsilon_{\mathrm{x}}+\varepsilon_{\mathrm{y}}}{2}+\frac{\varepsilon_{\mathrm{x}}-\varepsilon_{\mathrm{y}}}{2} \cos 2 \Theta .
$$

The strain on each rosette gauge can be represented in terms of the principal strain on $\mathrm{x}$ and $\mathrm{y}$ axis with corresponding rotation angles $\theta$, $\theta+60$ degrees, and $\theta+120$ degrees. Using the following strain transformation equations (Eq. 2a-2c), it is possible to determine the directionality of the strain applied. 


$$
\begin{aligned}
& \varepsilon_{\mathrm{s} 1}=\frac{\varepsilon_{\mathrm{x}}+\varepsilon_{\mathrm{y}}}{2}+\frac{\varepsilon_{\mathrm{x}}-\varepsilon_{\mathrm{y}}}{2} \cos 2 \Theta \\
& \varepsilon_{\mathrm{s} 2}=\frac{\varepsilon_{\mathrm{x}}+\varepsilon_{\mathrm{y}}}{2}+\frac{\varepsilon_{\mathrm{x}}-\varepsilon_{\mathrm{y}}}{2} \cos 2\left(\Theta+60^{\circ}\right) \\
& \varepsilon_{\mathrm{s} 3}=\frac{\varepsilon_{\mathrm{x}}+\varepsilon_{\mathrm{y}}}{2}+\frac{\varepsilon_{\mathrm{x}}-\varepsilon_{\mathrm{y}}}{2} \cos 2\left(\Theta+120^{\circ}\right)
\end{aligned}
$$

Therefore, by measuring the strain values of $\varepsilon_{s 1}, \varepsilon_{s 2}$, and $\varepsilon_{s 3}$, we could find the angle of $\theta$ with the following equation [18]:

$$
\theta=\left(\frac{1}{2}\right) \tan ^{-1}\left(\left(\sqrt{3}\left(\varepsilon_{s 2}-\varepsilon_{s 3}\right)\right) /\left(2 \varepsilon_{s 1}-\varepsilon_{s 2}-\varepsilon_{s 3}\right)\right)
$$

(a)

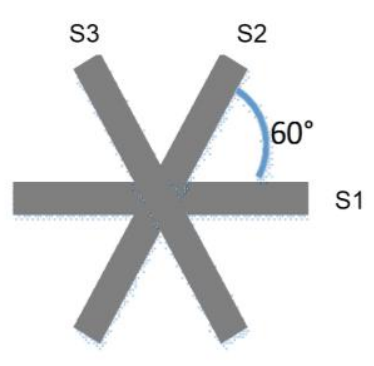

(b)

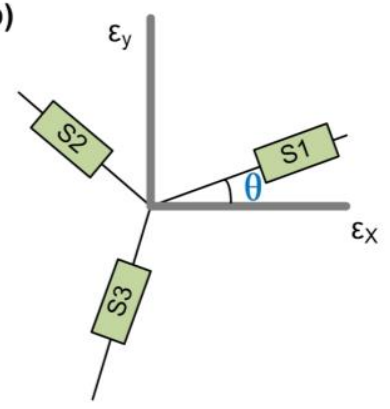

(c)

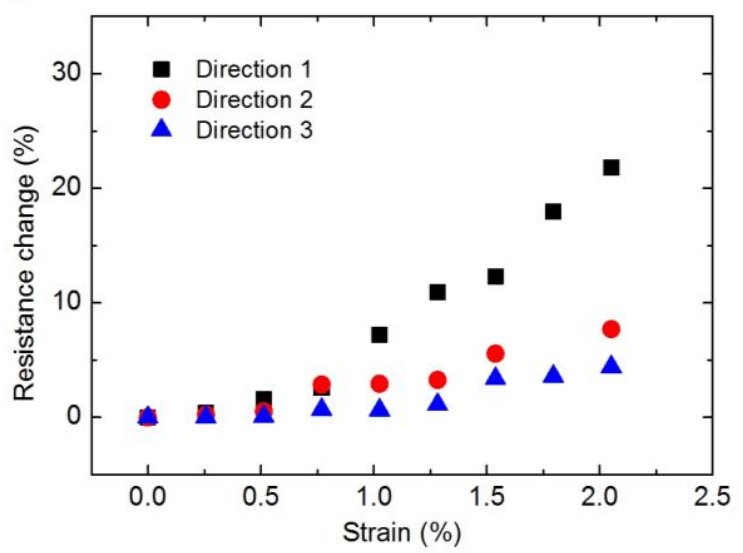

Figure 2 (a) S1, S2, and S3 graphene sensors stacked as a rosette gauge system. (b) Schematic representation of graphene strain sensor in rosette manner indicating the direction of principle axis (c) Variation of normalized resistance with respect to stretching up to $2.1 \%$ by a force applied in the direction $3^{\circ}$ to Direction 1 .

Fig. $5 \mathrm{c}$ demonstrates resistance responses of the three sensing elements in the rosette sensor to applied strains. The three sensing elements, $\mathrm{S}_{1}, \mathrm{~S}_{2}$, and $\mathrm{S}_{3}$ were arranged along the directions 1, 2 and 3 , respectively. When the external strain of $2.1 \%$ was applied to the device in the direction $3^{\circ}$ to Direction 1 , the resistance of $S_{1}, S_{2}$, and $\mathrm{S}_{3}$ was changed by $21.5 \%, 6.2 \%$ and $4.5 \%$, respectively. As discussed, by knowing the strain values of the three elements, the principal strain and its orientation with respect to the rosette axis could be estimated. Here, the principal strains $\varepsilon_{x}$ and $\varepsilon_{y}$ and their angle of orientation were calculated by inserting the three strain values of the three elements to Eq. (3). The value of $\theta$ was found to be $0.045(\mathrm{rad})$ or $2.604^{\circ}$ to Direction 1 .

The use of liquid metal interconnects in this graphene based strain sensor has several advantages. Firstly, the entire flexible structure allows the sensor to be installed in varies environments, such as the curved surface or moveable object. Also, its flexible structure decreases the fail rate caused by additional applied force on contact. Secondly, the liquid metal interconnectors keep the high flexibility around the detecting area, thus keeping the sensor's max sensitivity with minimal effect of less deformation due to solid contacting wires. To demonstrate these capabilities, liquid metal interconnects graphene film sensor were embedded inside concrete and bonded on human skin as two specific application examples.

Fig. 6 demonstrates the installation and testing of the unidirectional graphene strain sensor with liquid metal interconnect on concrete strain sensing application. The sensor was horizontally attached at the surface of a concrete cylinder. An external force was vertically loaded onto the cylinder. With a higher load, the concrete cylinder react an expansion in the horizontal plane. Therefore, the sensor felt a stretch strain while the concrete was expanded. The resistance of the sensor and the force load on the concrete were both recorded. The testing result indicates our sensors reacts in the similar pattern as the given load.

(a)

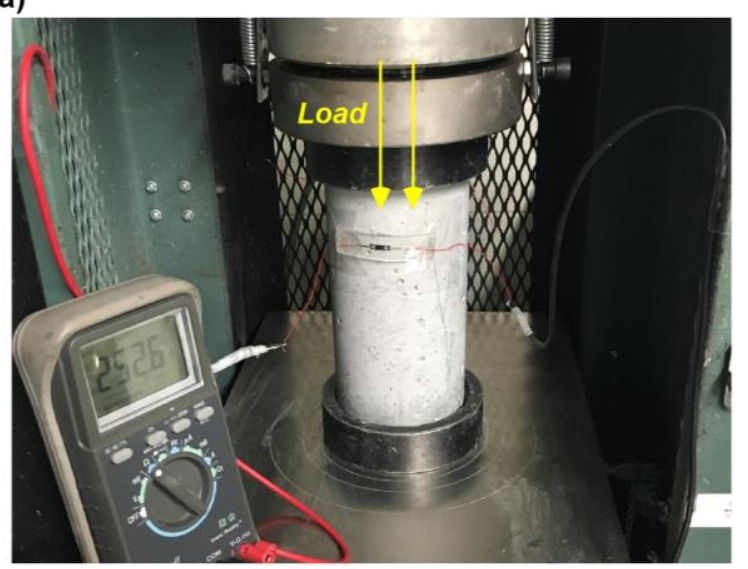

(b)

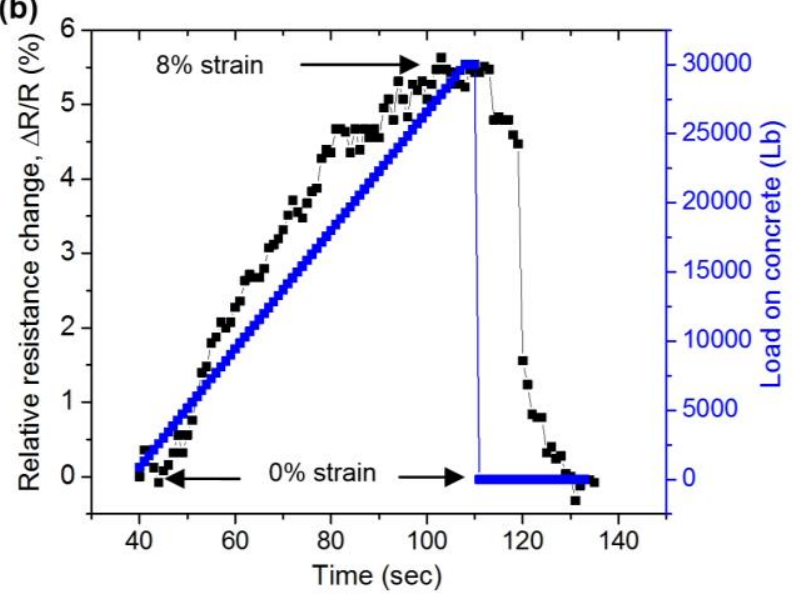

Figure 3: (a) Setup for monitoring strain changes of a concrete cylinder during loading and unloading processes. (b) Relative resistance change and load on the concrete cylinder over a period of time $(140 \mathrm{sec})$.

Fig. 7 provides the demonstration of the unidirectional sensor for tracking wrist motion. As the wrist bent up and down, the strain sensor changed its resistance. A larger bending degree led to a higher peak on resistance. The repeatability measurement $(\sim 200$ times) for this tactile sensor indicates that the device could provide good repeatability with a low relative standard deviation (RSD: $2.5 \%$ ) of peak resistance, which is believed due to good structural 
durability of the graphene sensing element and the liquid metal interconnectors.

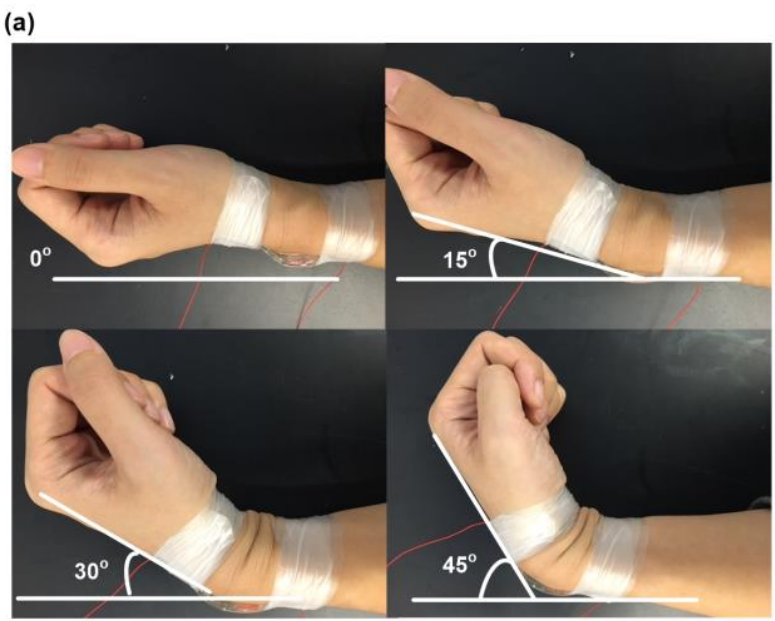

(b)

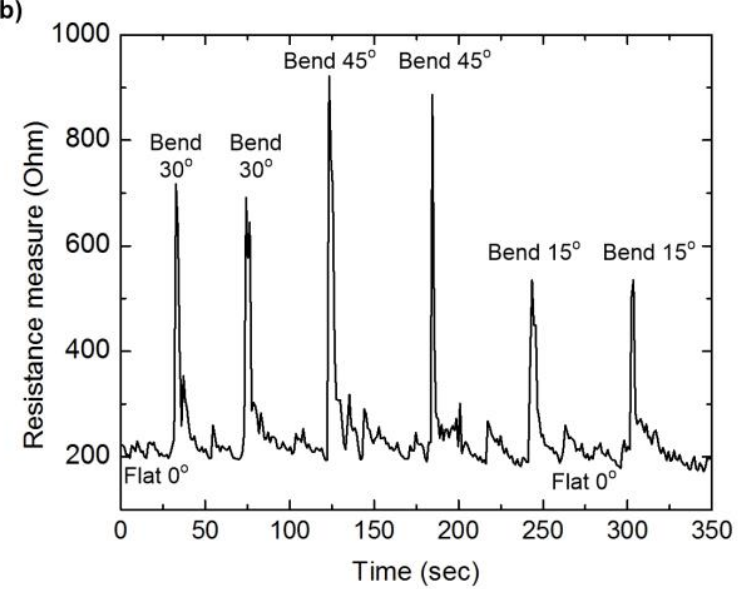

Figure 7: (a) Strain sensor bonded to waist to monitor waist motion. (b) Resistance response to waist motion. Resistance reflects the tensile strain and further indicates the different angles of waist movement.

\section{CONCLUSIONS}

This paper demonstrates a graphene based all flexible sensor which is built by: multilayer of graphene, liquid metal, and PMDS. We take advantage of graphene with its piezoresistive properties, which translates a mechanical displacement into an electrical signal. We take benefit from liquid metal interconnect for the better flexibility. Due its good repeatability and its linear strain response, this sensor will be useful for multiple applications, such as monitoring structural deformations on concrete, and tensile measurement on human skin.

\section{ACKNOWLEDGEMENTS}

This work was supported in part by the Iowa Department of Transportation, the Iowa Highway Research Board, and the U.S. National Science Foundation under grant ECCS-0954765.

\section{REFERENCES}

[1] J. Zhao, G. Zhang, and D. Shi, "Review of Graphene-based Strain Sensors", Chinese Physics B, 22, 057701 (2013).

[2] G. Zhang, J. Jing, J. Xu, X. Wu, W. Guo, and D. Yu , "Strain
Dependent Resistance in Chemical Vapor Deposition Grown Graphene", Applied Physics Letter, 99, 213107 (2011).

[3] M. Hempel, D. Nezich, J. Kong, and M. Hofmann, Nano Letter, "A Novel Class of Strain Gauges Based on Layered Percolative Films of 2D Materials", 12, 5714-5718 (2012).

[4] N.Chen, C. Tucker, C.Liu, S.H.Kim and D. Jones, "Flexible Multimodal Tactile Sensing System for Object Identification", IEEE Sensors 2006, EXCO, Daegu, Korea, 10/22-25/06 (2006), pp.563-566.

[5] N. B. Morley, J.Burris, L. C. Cadwallader, and M. D. Nornberg, "GaInSn Usage in the Research Laboratory", Review of Scientific Instruments, 79, 056107 (2008).

[6] H. Hu, K. Shaikh and C. Liu, "Super Flexible Sensor Skin using Liquid Metal as Interconnect", IEEE Sensors 2007, Atlanta, GA, 10/28-31/07 (2007), pp815-817.

[7] G.A. Hernandez, D. Martinez, C. Ellis, M. Palmer, and M. C. Hamilton, "Through Si Vias Using Liquid Metal Conductors for Re-workable 3D Electronics", Electronic Components and Technology Conference (ECTC), Las Vegas, NV, 05/28-31/13 (2013), pp1401-1406.

[8] B. Zhang, Q. Dong, C. E. Korman, Z. Li, and M. E. Zaghloul, "Flexible Packaging of Solid-state Integrated Circuit Chips with Elastomeric Microfluidics", Scientific Reports, 1098 (2013).

[9] S. Bae, Y. Lee, B. K. Sharma, H. Lee, J. Kim and J. Ahn, "Graphene-based Transparent Strain Sensor", Carbon, 51 236-242 (2013).

[10] Mase Geprge E. Schaum's outline of continuum mechanics. McGraw-Hill; 1969.

[11] H. Jiang, D. Khang, J. Song, Y. Sun, Y. Huang, J. Rogers. "Finite Deformation Mechanics in Buckled Thin Films on Compliant Supports", Proc Natl Acad Sci, 104(40):15607-12. (2007).

[12] C. Lee, X. Wei, J.W. Kysar, J. Hone, "Measurement of the Elastic Properties and Intrinsic Strength of Monolayer Graphene", Science, 321: 385-388 (2008).

[13] Y. Lee, S. Bae, H. Jang, S. Jang, S.-E. Zhu, S.H. Sim, et al., "Wafer-scale Synthesis and Transfer of Graphene Films", Nano Lett, 10: 490-493 (2010).

[14] X.-W. Fu, Z.-M. Liao, J.-X. Zhou, Y.-B. Zhou, H.-C. Wu, R. Zhang, et al., "Strain Dependent Resistance in Chemical Vapor Deposition Grown Graphene", Appl. Phys. Lett. 99: 213107 (2011).

[15] Y. Wang, R. Yang, Z. Shi, L. Zhang, D. Shi, E. Wang, et al., "Super-elastic Graphene Ripples for Flexible Strain Sensors", ACS Nano, 5: 3645-3650 (2011).

[16] O. Frank, G. Tsoukleri, I. Riaz, K. Papagelis, J. Parthenios, A.C. Ferrari, et al., "Development of a Universal Stress Sensor for Graphene and Carbon Fibres", Nat Commun, 2: 255 (2011).

[17] T. Yamada, Y. Hayamizu, Y. Yamamoto, Y. Yomogida, A. Izadi-Najafabadi, D.N. Futaba, et al., "A Stretchable Carbon Nanotube Strain Sensor for Human-motion Detection”, Nat Nanotechnol, 6: 296-301(2011).

[18] K. Loh, T.-C. Hou, J. Lynch, N. Kotov, "Carbon Nanotube Sensing Skins for Spatial Strain and Impact Damage Identification”, J Nondestr Eval, 28: 9-25 (2009).

\section{CONTACT}

*Liang Dong, tel: +1-515-294-0388; 1dong@iastaet.edu 